\title{
PKCa promotes the mesenchymal to amoeboid transition and increases cancer cell invasiveness
}

\author{
Katarína Vaškovičová1,2, Emilia Szabadosová', Vladimír Čermák1, Aneta Gandalovičová1, Lenka Kasalová', \\ Daniel Rösel ${ }^{1}$ and Jan Brábek ${ }^{1 *}$
}

\begin{abstract}
Background: The local invasion of tumor cells into the surrounding tissue is the first and most critical step of the metastatic cascade. Cells can invade either collectively, or individually. Individual cancer cell invasion can occur in the mesenchymal or amoeboid mode, which are mutually interchangeable. This plasticity of individual cancer cell invasiveness may represent an escape mechanism for invading cancer cells from anti-metastatic treatment.

Methods: To identify new signaling proteins involved in the plasticity of cancer cell invasiveness, we performed proteomic analysis of the amoeboid to mesenchymal transition with A375m2 melanoma cells in a 3D Matrigel matrix.

Results: In this screen we identified PKCa as an important protein for the maintenance of amoeboid morphology. We found that the activation of PKCa resulted in the mesenchymal-amoeboid transition of mesenchymal K2 and MDA-MB-231 cell lines. Consistently, PKCa inhibition led to the amoeboid-mesenchymal transition of amoeboid A375 m2 cells. Next, we showed that PKCa inhibition resulted in a considerable decrease in the invading abilities of all analyzed cancer cell lines.

Conclusions: Our results suggest that PKCa is an important protein for maintenance of the amoeboid morphology of cancer cells, and that downregulation of PKCa results in the amoeboid to mesenchymal transition. Our data also suggest that PKCa is important for both mesenchymal and amoeboid invasiveness, making it an attractive target for anti-metastatic therapies.
\end{abstract}

Keywords: Amoeboid, Mesenchymal, Plasticity, PKCa, Invasiveness, Metastasis

\section{Background}

The ability to form metastases is the most dangerous property that tumor cells can acquire. Cells of a primary tumor can disseminate throughout the body and potentially establish secondary tumors - metastases - in a process called the metastatic cascade (reviewed in [1]). The local invasion of tumor cells into the surrounding tissue is the first and most critical step of the metastatic cascade, and importantly, it determines the metastatic potential of many tumor cell types. Cells can invade through tissue and the extracellular matrix (ECM) either collectively, or individually. During collective invasion, the cell - cell adhesions between cells remain intact and cells migrate as a group in the form of strands, tubes,

\footnotetext{
* Correspondence: brabek@natur.cuni.cz

${ }^{1}$ Department of Cell Biology, Laboratory of Cancer Cell Invasion, Charles University in Prague, Prague, Czech Republic

Full list of author information is available at the end of the article
}

sheets or irregular masses [2-4]. Individual invasion is the invasion of single cells and can occur in mesenchymal or amoeboid mode (reviewed in [5,6]). The mesenchymal mode of invasion can be recognized by the typical fibroblast-like morphology of individually-invading cancer cells and also by their polarized character. At the leading edge, the cells generate actin rich structures, filopodia and lamellipodia, that trigger the cancer cell movement. Formation of filopodia and lamellipodia is regulated by the small GTPases Rac1 and Cdc42 [7,8]. Mesenchymal invasion is also dependent on local degradation of the ECM by degrading enzymes. The secretion of proteolytic enzymes is localized in actin-rich adhesion structures called invadopodia [9].

The morphology of amoeboid cells is typically round or ellipsoid in a 3D environment. Amoeboid cancer cell invasion is mediated by the contractions of cortical actin, which is regulated by the Rho/ROCK signaling pathway. 
Two types of Rho GTPase molecules, RhoA and RhoC, activate ROCK kinase. ROCK kinase phosphorylates MLCP (myosin light chain phosphatase) to inhibit its phosphatase function towards the myosin light chain (MLC), and ROCK therefore increases MLC [10-12]. To promote the effect, MLC2 is also directly phosphorylated by ROCK kinase. The phosphorylation of MLC leads to the generation of higher contractile forces by the actomyosin cortex, thus allowing the migration of cancer cells through ECM fibers independently of proteolytic degradation $[13,14]$.

Cancer cell invasion is a very complex and plastic process, and the mesenchymal and amoeboid modes of invasion are mutually interchangeable. Activation or inhibition of specific signaling cascades leading to a specific mode of invasion can cause a switch from one invasion mode to another (reviewed in $[5,6,15,16]$ ). It has been demonstrated that the mesenchymal-amoeboid transition (MAT) may be an escape mechanism in tumor cell invasion after the abolition of pericellular proteolysis [17]. The mechanisms of MAT or the amoeboid-mesenchymal transition (AMT) are, however, poorly understood. Only a limited number of studies describing the molecular mechanisms underlying MAT/AMT have been published so far (reviewed in [6]). In order to better understand the plasticity of individual cancer cell invasion, it is critical to identify other proteins involved in MAT and/or AMT.

To identify new signaling proteins involved in MAT/ AMT, we performed proteomic analysis of AMT with melanoma cells cultured in a 3D Matrigel matrix. Protein microarrays were chosen instead of gene expression microarrays because AMT and MAT are highly dynamic processes and thus are mostly defined by changes in posttranslational modifications of proteins and not in mRNA expression levels. To our knowledge, this is the first proteomic study of this kind performed with cells in a 3D matrix. We identified $\mathrm{PKC} \alpha$ as a protein important in the amoeboid mode of cancer cell invasion. Next, using biochemical and genetic approaches, we confirmed the role of $\mathrm{PKC} \alpha$ in regulating cancer cell morphology, the effectiveness of cancer cell invasiveness, and overall cancer cell plasticity.

\section{Methods}

\section{Cells, culture and material}

A375m2 melanoma cells were cultivated in full DMEM medium: DMEM (GIBCO) with $4500 \mathrm{mg} / \mathrm{l} \mathrm{L}$-glucose, L-glutamine, and pyruvate, supplemented with $10 \%$ fetal bovine serum (Sigma), $2 \%$ antibiotic-antimycotic (GIBCO) and 1\% MEM non-essential amino acids (GIBCO). MDAMB-231 breast cancer cells were maintained in full RPMI medium: RPMI (Gibco) supplemented with 10\% fetal bovine serum (Sigma), 2\% antibiotic-antimycotic (GIBCO) and 1\% MEM non-essential amino acids (GIBCO). K2 sarcoma cells were cultivated in in MEM with Hanks' salts supplemented with $10 \%$ bovine serum (ZVOS), $0.09 \%$ sodium bicarbonate, $0.12 \mathrm{~g} / \mathrm{L}$ sodium pyruvate, and $1 \mathrm{mmol} /$ $\mathrm{L}$ glutamine. All cell lines were kept at $37^{\circ} \mathrm{C}$ in a humidified atmosphere with $5 \% \mathrm{CO}_{2}$. Gö6976 - PKC $\alpha$ and PKC $\beta$ inhibitor, and PMA (phorbol 12-myristate 13-acetate) PKC activator were used at concentrations of $1 \mu \mathrm{M}$ and 162nM, respectively.

\section{DNA and RNA constructs and transfection}

Flag-tagged wt PKC $\alpha$, constitutive active form (with myristoylation sequence from $\mathrm{Src}$ ) and dominant-negative PKC $\alpha$ (K368R) in pCMV6 vector were all obtained from Addgene Inc. Silencer ${ }^{\circ}$ Select Validated siRNA against PKC $\alpha$ was obtained from Applied Biosystems and a final concentration of $10 \mathrm{nM}$ was used for transfections. All transfections were performed using jetPRIME ${ }^{\mathrm{Tm}}$ transfection kits (Polyplus Transfection) according to the manufacturer's instructions.

\section{Immunoblotting}

For 2D lysates, cells were cultured on plates with or without Y 27632 for 48 hours. then washed with phosphate buffered saline (PBS) and lysed in modified RIPA buffer $(0.15 \mathrm{M} \mathrm{NaCl} ; 50 \mathrm{mM}$ Tris $\mathrm{HCl}$ (pH 7.4); $1 \%$ Nonidet P 40; $0.1 \%$ SDS; $1 \%$ sodium deoxycholate; 5 mM EDTA; $50 \mathrm{mM} \mathrm{NaF}$ ).

For 3D lysates, cells were first embedded into $500 \mu \mathrm{l}$ of Matrigel solution (BD, MatrigelTM Basement Membrane Matrix Phenol Red Free) with or without Y 27632. After 24 hours, Matrigel was solubilized using Cell Recovery Solution (BD) for $30 \mathrm{~min}$ at $4^{\circ} \mathrm{C}$. Cells were washed with PBS and lysed in RIPA buffer.

Protein concentrations in the lysates were determined using the DC Protein Assay (Bio Rad). Protein lysates were diluted in Laemmli sample buffer $(0.35 \mathrm{M}$ Tris $\mathrm{HCl}$, pH 6.8; 10\% SDS; 40\% glycerol; 0.012\% bromphenol blue). For immunoblotting, samples were separated on 10\% SDS polyacrylamide gels and transferred onto nitrocellulose membranes. Nonspecific activity was blocked by incubating membranes for $1 \mathrm{~h}$ at $37^{\circ} \mathrm{C}$ in Tris buffered saline containing $4 \%$ bovine serum albumin. Membranes were then incubated overnight at $4^{\circ} \mathrm{C}$ with primary antibody, washed extensively with TTBS, and then incubated for $1 \mathrm{~h}$ at room temperature with HRP conjugated secondary antibody. After extensive washing in TTBS, the blots were developed using the LAS 1000 Single System (Leica). Monoclonal antibodies against FLAG anti-FLAG (F3165) and total $\mathrm{PKC} \alpha$ (P 4334) were from Sigma Aldrich Inc., PKC $\alpha$ pSer657 (sc-12356) and actin (sc 1616) were from Santa Cruz Biotechnology Inc., $\beta$-catenin antibody (610154) was from BD Transduction laboratories, p-cofilin Ser3 antibody (clone 77G2) was from Cell Signaling Technology. Secondary Antibodies GAR-HRP polyclonal (sc 2030) and 
DAG-HRP polyclonal (sc 2033) were from Santa Cruz Biotechnology Inc., and GAM-HRP polyclonal (35502) was from Thermo Fisher Scientific Inc.

\section{Protein microarrays}

$2.4 \times 10^{6}$ cells were embedded into $8 \mathrm{ml}$ of Matrigel (BD, MatrigelTM Basement Membrane Matrix Phenol Red Free) in the presence or absence of $50 \mu \mathrm{M}$ Y 27632. After 24 hours of incubation the Matrigel was solubilized with Cell Recovery Solution (BD) for $30 \mathrm{~min}$ at $4^{\circ} \mathrm{C}$. Cells were sedimented by centrifugation, washed in PBS and sent for analysis by Kinexus Bioinformatics Corporation (EXPANDED KINEX ${ }^{\mathrm{m}} 800$ ANTIBODY MICROARRAY SERVICE) on dry ice to determine changes in the expression and/or phosphorylation status of selected signaling proteins. Two experiments were performed in duplicates.

\section{Cell morphology assays in 3D collagen}

To analyze cell morphology of whole cell population in $3 \mathrm{D}$ collagen (in invasion assays only 10\% of cells reach deeper layers of 3D collagen), cells were trypsinized, washed in complete medium, counted, and then $10^{5}$ cells were mixed with $500 \mu \mathrm{l}$ of $3 \mathrm{mg} / \mathrm{ml}$ Collagen $\mathrm{R}$ (Serva) in complete medium. This suspension of cells in collagen $(500 \mu \mathrm{l})$ was loaded into a well of a 24 -well plate, the gel was allowed to polymerize at $37^{\circ} \mathrm{C}$ for $30 \mathrm{~min}$, and was then overlaid with complete medium. After 24 hours, the morphology of cells in 3D collagen was analyzed using a Nikon Eclipse TE2000-S microscope $(20 \times / 0,40$ HMC objective). Morphology of each cell was determined to be either elongated, intermediate or round. This was done on the basis of an elongation index. Elongation index was calculated as the length of the cell divided by the width. Cells whose elongation index was greater than 2 were considered elongated. Intermediate cells had an elongation index of 1,5-2; for rounded cells, the index was 1-1,5. Dividing cells were excluded from the analysis. Proportion of elongated, intermediate and rounded cells was calculated and expressed percentually. Three independent experiments (at least 300 cells per experiment) were analyzed for each condition. As the data have the form of counts in categories, the Pearson's Chi-squared test was used to reveal statistically significant differences.

\section{In vitro cell invasion assays in 3D collagen}

The 3D collagen invasion assay was analyzed as described previously [18]. Briefly, cell suspension $\left(1 \times 10^{5}\right.$ cells $\left./ \mathrm{ml}\right)$ was added on top of a collagen gel in a multiwell plate $(\mu-$ Slide Angiogenesis ibiTreat Microscopy Chamber (IBIDI)), and after 48 hours the level of invasion was measured. Pictures of 3D collagen cross-sections in the selected view were taken every $10 \mu \mathrm{m}$ using a Nikon-Eclipse TE2000-S (20×/0.40 HMC objective) and NIS-Elements software.
For each experiment, invasion was analyzed in 3 wells and average invasion depth was assessed in 6 fields of view per individual well. In order to compare individual experiments, the average invasion depth was normalized to that of untreated cells. In average the depth of at least $10 \mathrm{um}$ was achieved by 43 percent of K2 cells, 50 percent of MDA-MB-231 cells and 60 percent of A375m2 cells. Three independent experiments were analyzed for each condition. The significance of differences was analyzed with ANOVA followed by Tukey's honest significant difference test. The analysis was performed in R [19].

\section{Gelatin degradation assay}

Dry coverslips were coated with a thin layer of FITCconjugated gelatin and immediately overturned on a drop of $0.5 \%$ ice-cold glutaraldehyde in PBS for $15 \mathrm{~min}$ incubation in the dark. Coverslips were then transferred to a 12-well plate, with the coated side up and gently washed three times with PBS, incubated with sodium borohydride ( $5 \mathrm{mg} / \mathrm{ml}$ ) in PBS for $3 \mathrm{~min}$ and then finally washed with PBS. The cover slips were sterilized in $70 \%$ ethanol for $1 \mathrm{~min}$, dried for $10 \mathrm{~min}$ in a sterile hood and quenched in complete medium for $1 \mathrm{~h}$ at $37^{\circ} \mathrm{C}$. 48 hours after transfection, cells were plated onto prepared FITClabeled gelatin-coated coverslips. Overnight, cells were allowed to adhere and degrade gelatin and next day, cells were fixed and stained for actin. For actin staining, fluorescently labeled phalloidin (Alexa Fluor ${ }^{\circ} 405$ phalloidin (Invitrogen)) was used. Finally, coverslips were mounted on a mounting slide. Images for gelatin degradation assay were taken by fluorescent microscope Nikon Eclipse TE2000-S using (20×/0.40 HMC objective). Seven random fields were analyzed in each experiment using AnalyzeGelStack software (obtained from Prof. R. Buccione). Using this software, degradation area normalized to the number and area of cells (degradation area per cell) was measured. Values were normalized to value of control untreated cells. Confocal microscope Leica DM IRE2 was used for better cell visualization and taking representative pictures.

\section{Cell viability assay}

Cell viability was assessed by the trypan blue dye exclusion method. Briefly, cells were seeded into two $3.5 \mathrm{~mm}$ dishes, the next day $1 \mu \mathrm{M}$ Gö6976 was added to one of the dishes. After 48 hours cells were trypsinized and diluted 1:1 in $0.4 \%$ sterile filtered trypan blue solution. The cells were incubated in the trypan blue solution for 5 minutes at room temperature and then transferred onto a haemocytometer and counted under a light microscope. Viable cells appeared bright, dead cells were blue. Three independent experiments were performed, each time both sides of the haemocytometer were counted twice for both conditions. To assess the statistical significance of the differences, the Pearson's Chi-squared test was performed. 


\section{Results}

Protein microarray analysis reveals the down-regulation of PKCa phosphorylation in highly metastatic amoeboid A375m 2 melanoma cells after AMT

To identify new proteins involved in amoeboid invasiveness and the AMT, we analyzed proteomic profiles of cells before and after AMT. We used amoeboid melanoma A375m2 cells that were cultivated in 3D Matrigel and either induced AMT with the ROCK kinase inhibitor Y-27632 or left the cells untreated. We observed $100 \%$ rounded A375m 2 cells in Matrigel before addition of Y-27632. After the Y-27632 the number of rounded cells was decreased to $43 \%$. 31\% were elongated and $26 \%$ exhibited intermediate morphology (see also Additional file 1: Figure S1). Cell lysates from both untreated and treated cells were analyzed using protein microarray analysis (Kinex). 30 proteins showed significant changes in expression levels between cells cultivated without and with Y-27632. Moreover, 11 proteins exhibited significant changes in the phosphorylation of analyzed sites (Table 1). Among these proteins, we selected several candidates with potential connections to AMT-related signaling for further verification. These included $\beta$-catenin (increased expression after AMT), cofilin-2 (increase Ser3 phosphorylation after AMT) and PKC $\alpha$ (decreased Ser657 phosphorylation after AMT). For all the three selected proteins we confirmed the trends in changes of their phosphorylation (cofilin-2, PKC $\alpha$ ) or expression ( $\beta$-catenin) status after AMT (Figure 1). Interestingly, the changes were only seen when Y-27632 was administered in the 3D Matrigel and not in 2D conditions (Figure 1), suggesting that these changes are in fact specific for AMT. Since we were most interested in proteins with potential involvement in amoeboid invasiveness, in our next experiments we concentrated on PKC $\alpha$ which was found to be downregulated after AMT.

Inhibition of PKCa results in AMT in amoeboid cells, PKC activation results in MAT in mesenchymal cells

The phosphorylation of PKC $\alpha$ at Ser657 is associated with increased stability of the kinase [20,21]. Our protein microarray analysis thus suggests that PKC $\alpha$ activation could possibly be involved in maintenance of the amoeboid morphology of melanoma A375m 2 cells. To investigate the possible role of $\mathrm{PKC} \alpha$ in regulating cell morphology, we analyzed the morphology of selected mesenchymal and amoeboid cell lines in 3D collagen in the presence and absence of a PKC $\alpha$ activator or inhibitor. The A375m 2 human melanoma cell line, K2 rat sarcoma cell line and MDA-MB-231 human breast cancer cell line were used. Cells were seeded into 3D collagen matrix and the morphology of cells was observed after 48 hours of incubation. As a PKC $\alpha$ activator, phorbol 12-myristate 13-acetate (PMA) was used at a final concentration of 162nM. PMA
Table 1 Proteins with differential expression/ phosphorylation after AMT in A375m2 cells (Kinexus)

\begin{tabular}{|c|c|c|c|}
\hline Protein & Phosphosite (human) & Refseq & Fold change \\
\hline IKK alpha & Pan-specific & NP_001269 & 6.7 \\
\hline CDK7 & Pan-specific & NP_001790 & 5.2 \\
\hline ASK1 (MAP3K5) & Pan-specific & NP_005914 & 3.2 \\
\hline STAT1a/b & Pan-specific & NP_009330 & 3.2 \\
\hline Catenin beta & Pan-specific & NP_001895 & 2.4 \\
\hline MEK6 (MAP2K6) & S207 & NP_002749 & 2.3 \\
\hline Erk2 & Pan-specific & NP_002736 & 2.3 \\
\hline Cofilin 2 & S3 & NP_068733 & 2.3 \\
\hline CASP4 & Pan-specific & NP_001216 & 2.1 \\
\hline Hsp90 & Pan-specific & NP_005339 & -26.4 \\
\hline$J N K 1 / 2 / 3$ & Pan-specific & NP_002741 & -22.6 \\
\hline Bid & Pan-specific & NP_001187 & -15.2 \\
\hline CDK1 (CDC2) & Pan-specific & NP_001777 & -9.6 \\
\hline PERP & Pan-specific & NP_071404 & -8.7 \\
\hline $\mathrm{Hsc} 70$ & Pan-specific & NP_006588 & -6.1 \\
\hline JNK2 & Pan-specific & NP_002744 & -5.2 \\
\hline APG1 & Pan-specific & NP_055093 & -5.0 \\
\hline PDK1 & Pan-specific & NP_002604 & -4.3 \\
\hline $\mathrm{HO} 1$ & Pan-specific & NP_002124 & -3.8 \\
\hline PLC gamma 1 & Y783 & NP_877963.1 & -3.6 \\
\hline CaMK1d & Pan-specific & NP_003647 & -3.5 \\
\hline MEK4 (MAP2K4) & Pan-specific & NP_003001 & -3.3 \\
\hline PKC alpha & S657 & NP_002728 & -3.2 \\
\hline IRAK2 & Pan-specific & NP_001561 & -3.2 \\
\hline Mnk2 & Pan-specific & NP_060042 & -3.1 \\
\hline p35 & Pan-specific & NP_003876 & -3.0 \\
\hline FAK & Y577 & NP_005598 & -3.0 \\
\hline BRCA1 & $\mathrm{S} 1497$ & NP_009225 & -3.0 \\
\hline BRCA1 & $\mathrm{S} 1423$ & NP_009225 & -2.9 \\
\hline 4E-BP1 & T45 & NP_004086 & -2.6 \\
\hline Bak & Pan-specific & NP_001179 & -2.5 \\
\hline MEK1 (MAP2K1) & T385 & NP_002746 & -2.5 \\
\hline MEK5 (MAP2K5) & Pan-specific & NP_660143 & -2.4 \\
\hline TrkA & Pan-specific & NP_002520 & -2.4 \\
\hline Pax2 & S394 & NP_000269.2 & -2.4 \\
\hline STAT3 & Pan-specific & NP_003141 & -2.3 \\
\hline MEK7 (MAP2K7) & Pan-specific & NP_005034 & -2.3 \\
\hline PKC epsilon & S729 & NP_005391 & -2.3 \\
\hline PKBb (Akt2) & Pan-specific & NP_001617 & -2.1 \\
\hline GCK & Pan-specific & NP_004570 & -2.1 \\
\hline p38a MAPK & Pan-specific & NP_001306 & -2.1 \\
\hline
\end{tabular}

Antibody microarray (Kinex) screen of A375m2 cells in AMT. Cells in 3D culture were treated with ROCK inhibitor and analyzed after 24 hours. Signal values from duplicate samples were tested for significant differences with Student's t-test followed by $\mathrm{p}$-value correction for multiple testing (Benjamini-Hochberg). Proteins that showed at least two-fold change and FDR $<0.3$ are shown here, complete data are available in supplementary information. Proteins discussed in the text are in boldface. 


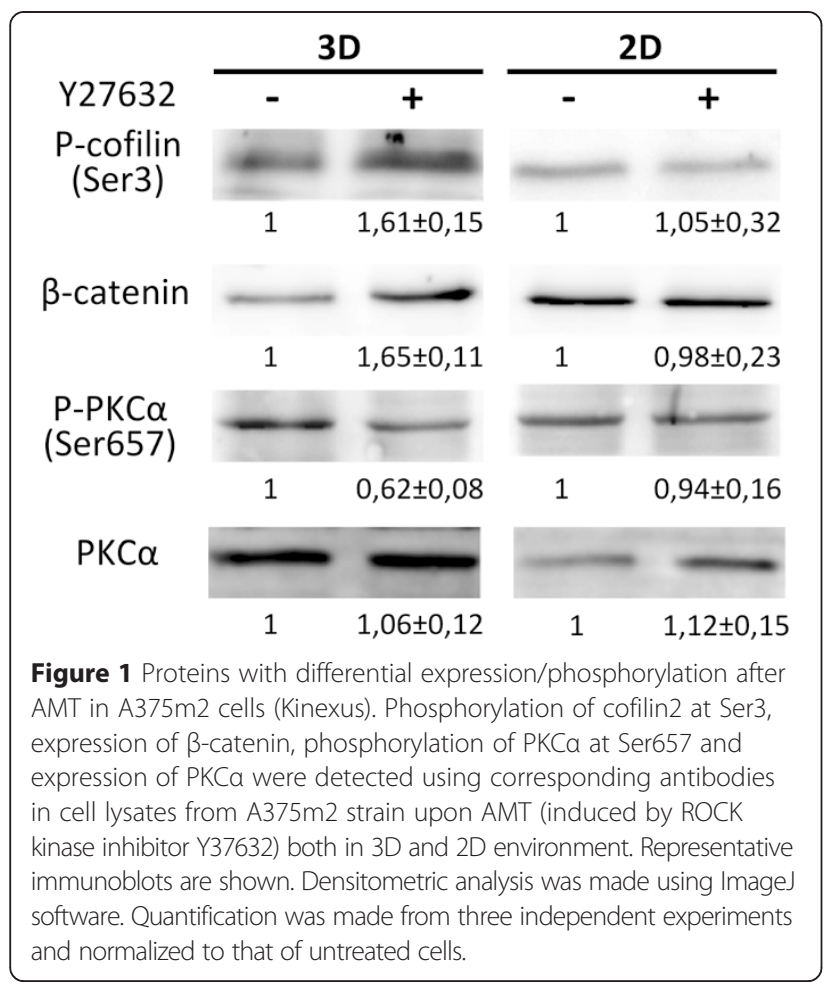

is not a PKC $\alpha$ specific activator, but is able to activate all PKC isoforms. As a PKC $\alpha$ inhibitor, Gö6976 was used at a $1 \mu \mathrm{M}$ concentration. Gö6976 is a PKC $\alpha / P K C \beta$ specific inhibitor. However, proteomic analysis did not show any changes in other PKC isoforms than PKC $\alpha$, so the activator and particularly the inhibitor were considered to be sufficiently specific for our experiments.

Analysis of the cellular morphology in 3D collagen confirmed that A375m2 cells are primarily amoeboid (rounded cell morphology) and K2 and MDA-MB-231 cells are primarily mesenchymal (elongated cell morphology) (Figure 2, Additional file 2: Figure S2). Upon PMA treatment, the morphology of $\mathrm{K} 2$ and MDA-MB231 cells shifted from mesenchymal towards amoeboid. For K2 cells, the proportion of rounded cells was increased approximately 4 fold, and for MDA-MB-231 cells a 3 fold increase was observed (Figure 2, Additional file 2: Figure S2). In both cases, the number of elongated cells decreased proportionally. This strongly suggests the occurrence of the MAT. PMA did not have a significant effect on A375m2 cells as these cells were already of predominantly rounded morphology. In contrast, treatment with the PKC $\alpha$ inhibitor Gö6976 resulted in the AMT of A375m 2 cells, manifested by a 2.5 fold increase in the proportion of elongated cells. Simultaneously, the proportion of rounded cells decreased. Gö6976 did not greatly affect the morphology of $\mathrm{K} 2$ and MDA-MB-231 cells as these cells were already of mainly elongated morphology (Figure 2, Additional file 3: Figure S3).

\section{Inhibition of PKCa results in decreased cancer cell invasiveness}

Next, the invasivity of cells in vitro was analyzed to determine the effect of PKC $\alpha$ on the capacity of cells to invade $3 \mathrm{D}$ collagen. Incubation of cells with the PKC activator PMA did not significantly change the cell invasion potential of any of the three cell lines examined. In contrast, treatment with the PKC $\alpha / \beta$ inhibitor Gö6976 resulted in a mild but significant decrease in the invasiveness of all three cell lines (Figure 3 ). This decrease was 1.3 fold in the case of $\mathrm{K} 2$ cells, 1.5 fold in the case of MDA-MB-231 cells, and 1.4 fold in the case of A $375 \mathrm{~m} 2$ cells. To exclude the possibility that the decreased invasiveness is caused by cytotoxic effect of PKC alpha inhibition, we have analyzed the effect of Gö6976 on cell survival of MDA-MB-231 cells. We found no significant effect of the inhibitor at the concentration used in our assays $(1.7 \%$ and $3.3 \%$ dead cells in control and treated cells, respectively with chi-squared test p-value 0.197 ).

\section{Overexpression or activation of PKCa promotes MAT in mesenchymal cells, dominant-negative PKCa promotes AMT in amoeboid cells}

To further confirm the role of PKC $\alpha$ in amoeboid invasiveness and AMT/MAT we decided to validate the effects of the PKC inhibitor and activator using a genetic approach. In all three cell lines we overexpressed wt PKC $\alpha$ or dominant-negative PKC $\alpha$ (K368R) to achieve activation and inhibition of $\mathrm{PKC} \alpha$, respectively. In transfected cells, the expression of each PKC $\alpha$ variant was demonstrated using an immunoblot (Additional file 3: Figure S3A). Surprisingly, the expression of dominantnegative PKC $\alpha$ was much lower than that of wt PKC $\alpha$, possibly due to negative selection. The activation status of the variants was verified with a phospho-specific antibody against Thr497 in the activation loop of $\mathrm{PKC} \alpha$ (Additional file 3: Figure S3A).

To verify the possible role of $\mathrm{PKC} \alpha$ in regulating AMT/MAT, we performed morphology assays in 3D collagen with the A375m2 human melanoma cell line, K2 rat sarcoma cell line and MDA-MB-231 human breast cancer cell line transfected with PKC $\alpha$ variants. Upon the overexpression of wt PKC $\alpha, \mathrm{K} 2$ and MDA-MB-231 cells changed their morphology towards amoeboid. For both cell lines, the proportion of rounded cells was increased approximately 1.7 fold (Figure 4, Additional file 4: Figure S4) and the number of elongated cells decreased proportionally. This strongly suggests the occurrence of MAT. The expression of wt PKC $\alpha$ did not have significant effect on A375m2 cells as these cells were already of predominantly rounded morphology. In contrast, the expression of dominant-negative PKC $\alpha$ resulted in the AMT of A $375 \mathrm{~m} 2$ cells, manifested by a 2 fold increase in the proportion of elongated cells. Simultaneously, the proportion 

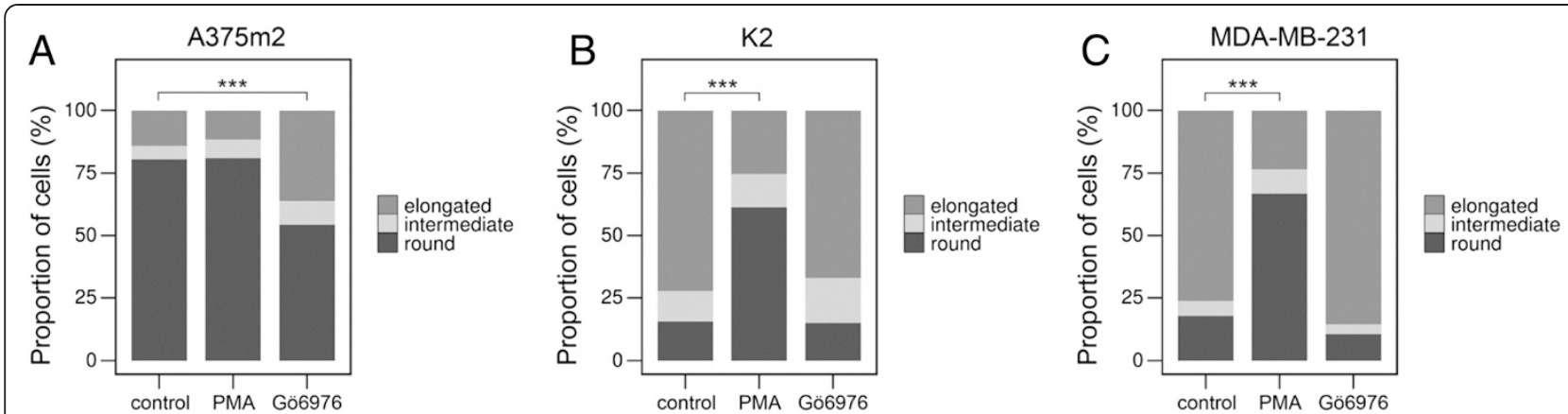

Figure 2 Inhibition of PKCa results in AMT in amoeboid cells, PKC activation results in MAT in mesenchymal cells. Cell morphology analysis of A375m2 (A), K2 (B) and MDA-MB-231 (C) strains upon PKC activator PMA or PKCa/ $\beta$ inhibitor Gö6976 treatment. Cells were incubated with PMA $(162 n M)$ or Gö6976 (1 HM) for 48 hours in 3D collagen and then analyzed using NIS Elements software (Nikon). Cells were classified according to the elongation index as rounded, intermediate or elongated (see Methods section). Statistical significance (marked by asterisks) was evaluated using Pearson's Chi-squared test. Representative results are shown out of minimum three independent experiments.

of rounded cells decreased. The expression of dominantnegative PKCa did not greatly affect the morphology of K2 and MDA-MB-231 cells as these cells were already of mainly elongated morphology (Figure 4, Additional file 4: Figure S4).

\section{Expression of dominant-negative PKCa results in decreased cancer cell invasiveness}

Next, the invasivity of cells in vitro was analyzed to determine the effect of PKC $\alpha$ variants on the capacity of cells to invade 3D collagen. Again, K2, A375m2 and MDAMB-231 cell lines transfected with wt or dominantnegative PKC $\alpha$ were used. The expression of wt PKC $\alpha$ resulted in a slight, but not significant decrease of invasiveness in mesenchymal cell lines and also a non-significant increase of invasiveness in amoeboid A375m2 cells. More importantly, the expression of dominant-negative PKC $\alpha$ resulted in a significant decrease of invasiveness in all investigated cancer cell lines (Figure 5). These results suggest that $\mathrm{PKC} \alpha$ is important for effective cancer cell invasiveness irrespective of the type of cancer cell invasion.

\section{siRNA-mediated downregulation of PKCa results in AMT in A375m2 amoeboid cells}

To further confirm the specific effect of PKC $\alpha$ isoform on the cell morphology and invasiveness of amoeboid cancer cells, specific downregulation of PKC $\alpha$ expression in $\mathrm{A} 375 \mathrm{~m} 2$ cells was performed using siRNA against PKC $\alpha$. In comparison to control and mock-transfected cells, total PKC $\alpha$ expression level in siRNA treated cells was significantly lower (Additional file 3: Figure S3B). In agreement with the effect of the PKC $\alpha$ inhibitor and
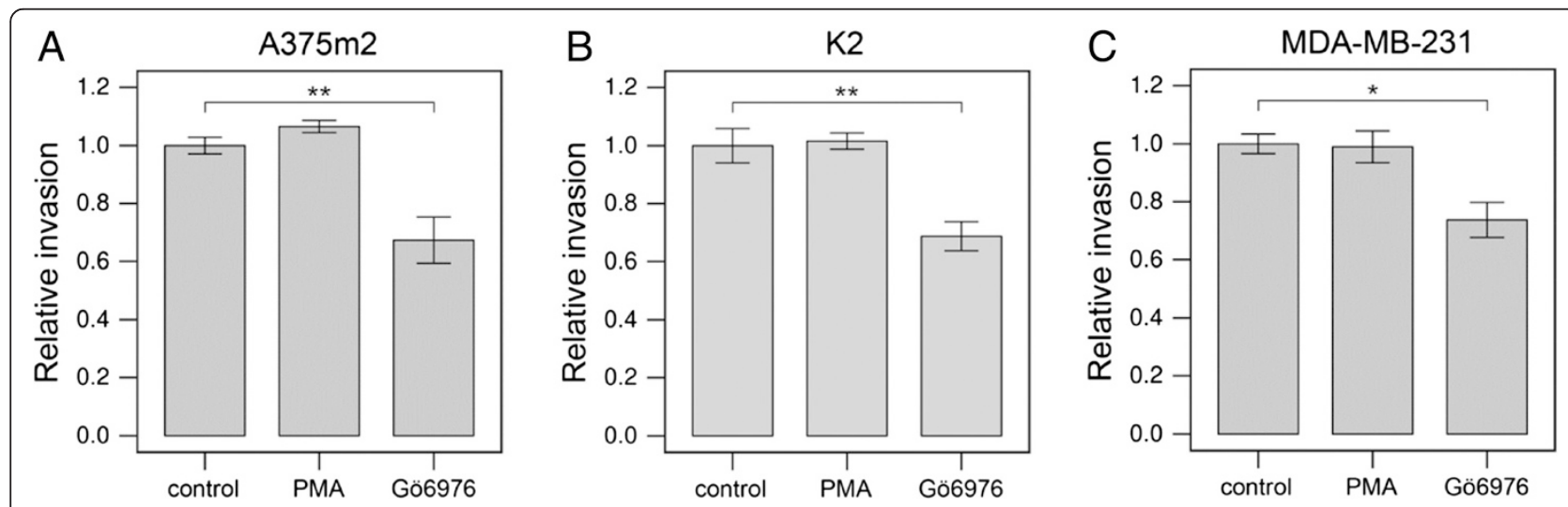

Figure 3 Inhibition of PKCa results in decreased invasiveness of cancer cells. Invasion analysis of A375m2 (A), K2 (B) and MDA-MB-231 (C) strains upon PKC activator PMA or PKCa/ $\beta$ inhibitor Gö6976 treatment. Cells were plated onto 3D collagen and let to invade it for 48 hours in the presence of PMA (162nM) or Gö6976 (1 M). 3D collagen cross-sections were analyzed using NIS Elements software (Nikon) and average invasion depth was calculated and normalized to that of untreated cells. The invasion depth in each individual field of view was assessed as sum of invasion depths of individual cells divided by the total number of cells in the respective field of view (including cells on the top of collagen). Statistical significance (marked by asterisks) was evaluated using ANOVA followed by Tukey's honest significant difference test. 

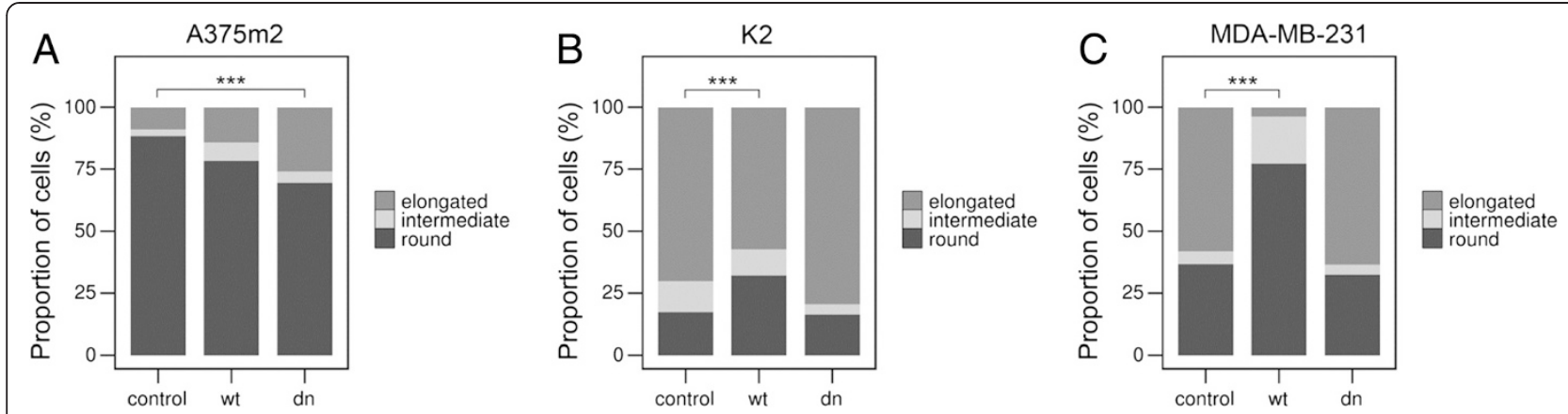

Figure 4 Dominant-negative PKCa promotes AMT in amoeboid cells, overexpression of PKCa promotes MAT in mesenchymal cells. Cell morphology analysis of A375m2 (A), K2 (B) and MDA-MB-231 (C) strains overexpressing wt PKCa or expressing dominant-negative PKCa. Cells were transfected with pCMV6 vector expressing either wt PKCa or dominant-negative PKCa and their morphology was observed after 48 hours in 3D collagen. Cells were analyzed using NIS Elements software (Nikon). Cells were classified according to the elongation index as rounded, intermediate or elongated (see Methods section). Statistical significance (marked by asterisk) was evaluated using Pearson's Chi-squared test. Representative results are shown out of minimum three independent experiments.

expression of dominant-negative PKC $\alpha$, siRNA treated cells exhibited a more mesenchymal morphology profile than control and mock-transfected cells, suggesting the induction of AMT. The proportion of elongated cells increased 2 fold, and there was a minor increase in the proportion of intermediate cells as well. Consistently, the proportion of rounded cells decreased (Figure 6A, Additional file 5: Figure S5). The amoeboid to mesenchymal transition was also manifested by elevated ability of siRNA treated A375m2 cells to degrade gelatin (Additional file 6: Figure S6).

In vitro invasion analysis of siRNA transfected cells showed that $\mathrm{PKC} \alpha$ downregulation reduced the cell invasivity of A375m2 cells in 3D collagen (Figure 6B).
Compared to mock-transfected cells, the ability of siRNA treated cells to invade into 3D collagen was reduced 1.5 fold, and this reduction was even higher (2.5 fold) compared to untreated cells. This is consistent with previous experiments where PKC $\alpha$ inhibition also resulted in a significant decrease in cell invasion potential. Together, these results further confirm the important role of $\mathrm{PKC} \alpha$ in amoeboid invasiveness.

\section{Discussion}

PKC $\alpha$ is a very important protein regulating many signaling pathways in cells, such as proliferation, differentiation, apoptosis and tumorigenesis (reviewed in [22]), and its deregulation is involved in many diseases (reviewed in [23]).

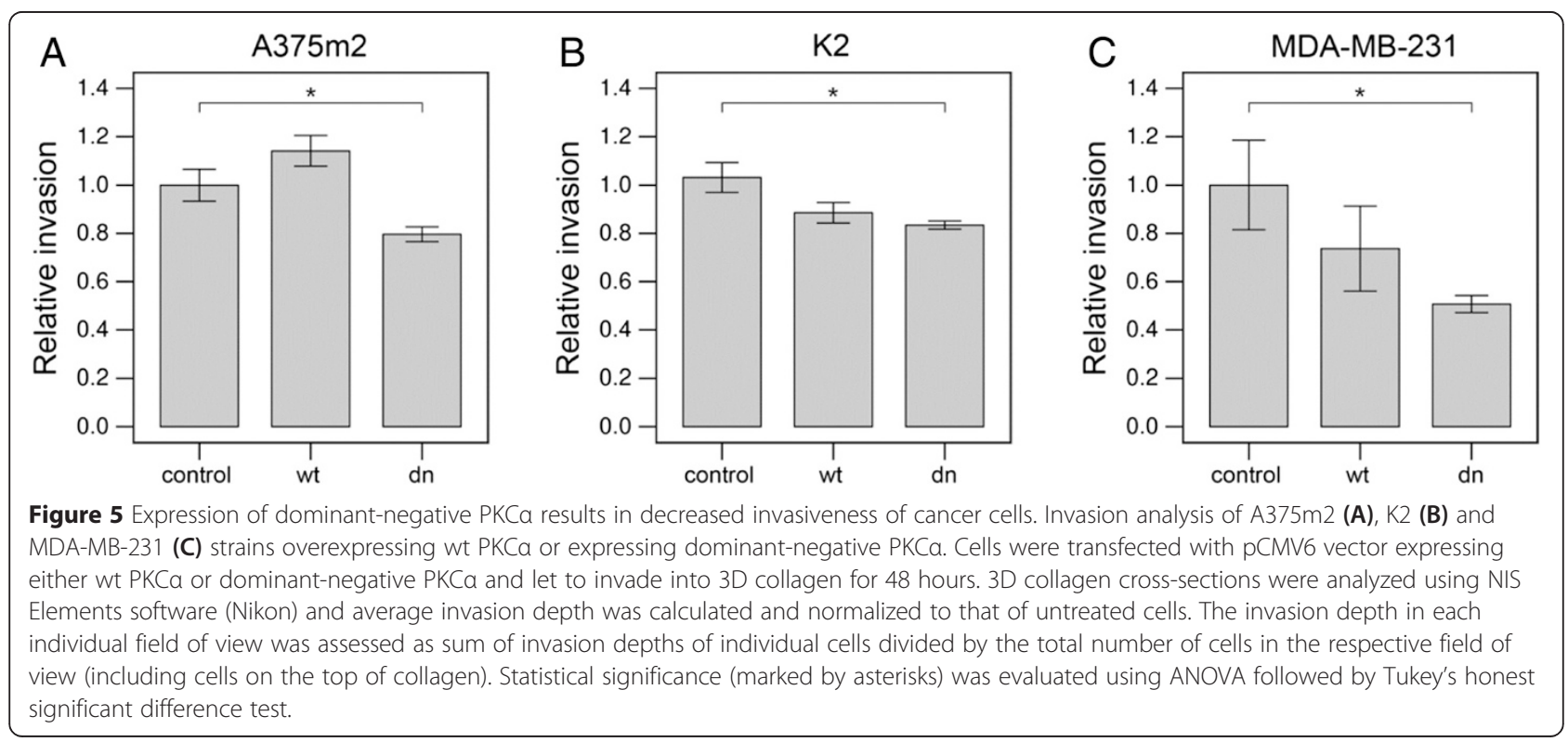



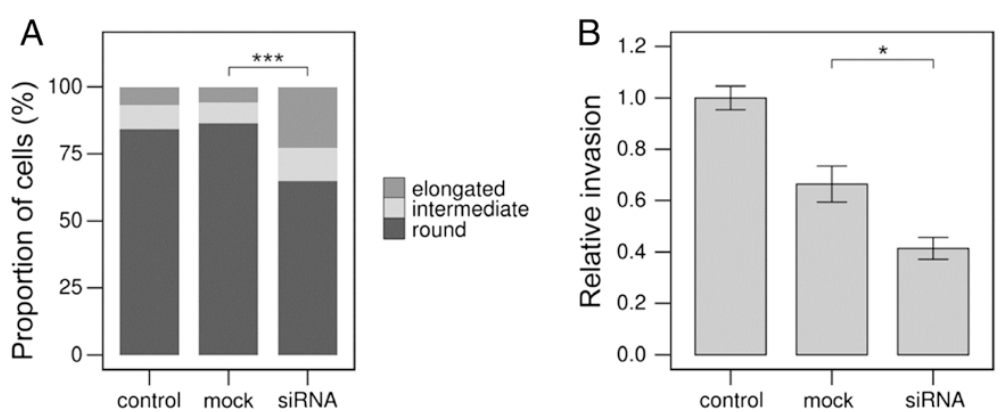

Figure 6 siRNA-mediated downregulation of PKCa results in AMT in A375m2 amoeboid cells. A) Cell morphology analysis of A375m2 strain transfected with siRNA against PKCa. Cells were transfected with siRNA and their morphology was observed after 48 hours in 3D collagen. Cells were analyzed using NIS Elements software (Nikon). Cells were classified according to the elongation index as rounded, intermediate or elongated (see Methods section). Statistical significance (marked by asterisk) was evaluated using Pearson's Chi-squared test. Representative result is shown out of three independent experiments. B) Cell invasion analysis of A375m2 strain transfected with siRNA against PKCa. Cells were transfected with siRNA and let to invade into 3D collagen for 48 hours. 3D collagen cross-sections were analyzed using NIS Elements software (Nikon) and average invasion depth was calculated and normalized to that of untreated cells The invasion depth in each individual field of view was assessed as sum of invasion depths of individual cells divided by the total number of cells in the respective field of view (including cells on the top of collagen). Statistical significance (marked by asterisks) was evaluated using ANOVA followed by Tukey's honest significant difference test.

PKC $\alpha$ has also been shown to regulate cell migration and cancer cell invasion [24-29]. Proteomic analysis of amoeboid A375m2 cells grown in 3D Matrigel revealed that phosphorylation of $\mathrm{PKC} \alpha$ at Ser657 is reduced upon a ROCK kinase inhibitor Y-27632 mediated induction of AMT. Importantly, we found that the observed changes in the phosphorylation of PKC $\alpha$ at Ser657 and two additional candidate markers of AMT occur only in 3D Matrigel and not in 2D conditions. These results confirm the necessity of performing AMT analyses in the $3 \mathrm{D}$ environment.

In addition to decreased levels of PKC $\alpha$ phosphorylation at Ser657, the phosphoproteomics screen revealed a significant decrease in the phosphorylation of a PKC $\alpha$ upstream activator, PLC 1 on Tyr783 (Table 1). This suggests that treatment with the ROCK inhibitor Y27632 affects signaling leading to the activation of PKC $\alpha$, rather than directly inhibiting PKC $\alpha$.

Ser657 is located in the hydrophobic region of PKC $\alpha$ and its phosphorylation controls the accumulation of active PKC $\alpha$ and prevents its down-regulation $[20,21]$. The phosphoproteomics screen data thus suggested that by affecting the level of PKC $\alpha$ activation we could induce AMT or MAT in cancer cells. To confirm this, we analyzed the effect of PKC $\alpha$ inhibition and activation on the morphology of amoeboid A375m2 and two mesenchymal K2 and MDA-MB-231 cell lines. This morphology analysis revealed that the level of PKC $\alpha$ activation is indeed important for the regulation of cell morphology in the 3D environment. PKC $\alpha$ activation resulted in MAT in both mesenchymal cell lines tested and, correspondingly, PKC $\alpha$ inhibition or downregulation resulted in AMT in amoeboid A375m2 cells.
Next, in vitro invasivity assays into 3D collagen were performed to elucidate the effect of $\mathrm{PKC} \alpha$ directly on cell invasiveness. Although PKC $\alpha$ activation did not significantly influence the invasivity of cells, PKC $\alpha$ inhibition resulted in a considerable decrease in the invading abilities of all three cell lines used (K2, A $375 \mathrm{~m} 2$ and MDA-MB-231). These results suggest that a proper level of $\mathrm{PKC} \alpha$ activation is necessary for effective invasiveness of both amoeboid and mesenchymal cancer cell lines. This is consistent with previous results that have shown that PKC $\alpha$ regulates cell invasion and migration in several cancer cell lines. Inhibition of PKC $\alpha$ has decreased the invasion of urinary bladder carcinoma [30], colon carcinoma [31], renal cell carcinoma [32], breast carcinoma [33], multiple myeloma [34], glioma [35], breast cancer [36] and endometrial cancer cells [37]. PKC $\alpha$ activation and overexpression have been shown to increase cancer cell migration $[31,33,34,36]$. Consistently, PKC $\alpha$ knockdown leads to a decrease in cancer cell invasion $[31,36,37]$. Remarkably, PKC $\alpha$ was shown to be a marker of aggressiveness in breast cancer, as patients with $\mathrm{PKC} \alpha$-positive tumors exhibited poorer prognosis than patients with $\mathrm{PKC} \alpha$-negative tumors [36].

Since PKC alpha was shown to be involved in cancer cell survival (e.g. [38]), we had to rule out the possibility the observed decrease of invasiveness could be at least in part caused by a weak cytotoxic effect of PKC $\alpha$ inhibition. We found that under our conditions, PKC $\alpha$ inhibition does not have detectable cytotoxic effect.

Interestingly, the PKC $\alpha$ overexpression resulted in possibly decreased invasion (but not significant) in mesenchymal cell lines and increased invasion (also not significant) in amoeboid cell line, suggesting that for mesenchymal 
invasiveness endogenous level of PKC $\alpha$ activity could be optimal and further increase of PKC $\alpha$ expression may not result in increased invasion capability. Also it has to be taken into account that amoeboid migration is faster particularly under non-restrictive conditions [39]. In dense $3 \mathrm{D}$ collagen gels the size of the pores is limiting for nonproteolytic migration and under such conditions, tunnels formed by the mesenchymal cells could be a more effective strategy, especially in cells which are originally mesenchymal (K2) or intermediate (MDA-MB-231).

Together, these results suggest a possible role of $\mathrm{PKC} \alpha$ in the amoeboid invasion of cancer cells. The setup of our proteomic screen and the effects of PKC $\alpha$ activity on amoeboid cancer cells suggest that PKC $\alpha$ might activate the small GTPase RhoA or ROCK kinase. The Rho/ ROCK signaling pathway controls phosphorylation of the myosin light chain and mediates the enhanced actomyosin contractility that is a critical feature of amoeboid cells $[10,11,14]$. However, it is likely that PKC $\alpha$ does not phosphorylate RhoA or ROCK directly; rather, $\mathrm{PKC} \alpha$ might phosphorylate their upstream regulators.

Indeed, there is evidence in the literature of $\mathrm{PKC} \alpha$ regulating the Rho/ROCK signaling pathway through upstream regulators, together with their relevance to cell adhesion and cytoskeletal reorganization. Earlier results showed that PKCa can be activated upon thrombin treatment and can enhance RhoA activity in endothelial cells, therefore mediating cell contraction $[40,41]$. PKC $\alpha$ phosphorylates p115RhoGEF, a specific Rho guanine nucleotide exchange factor, and increases its activity [42]. Consequently, RhoA activity is also increased and this is connected to the regulation of stress fiber formation and cell contraction [42]. One of the PKC $\alpha$ substrates is also RhoGDI $\alpha$ [43]. RhoGDI $\alpha$ is a cytosolic protein that binds the GDP-bound small RhoGTPases RhoA, Rac1 and Cdc42, and prevents them from the exchange of GDP to GTP [44]. PKC $\alpha$ phosphorylates RhoGDI $\alpha$ at Ser34 to reduce its affinity for RhoA (but not for Rac1 or Cdc42) [43]. So, RhoA can be activated by GTP binding and downstream signaling is enhanced, as was observed by a loss of focal adhesions [40]. Also, phosphatidylinositol bisphosphate is needed for RhoGDI $\alpha$ phosphorylation by $\mathrm{PKC} \alpha$; it is probably required for proper PKC $\alpha$ localization and orientation in the membrane [43]. Moreover, $\alpha v \beta 3$ integrin and syndecan4-mediated PKC $\alpha$ activation enhances RhoA activity to form focal adhesions and maintain stress fibers $[45,46]$.

More recently, results that connect PKC $\alpha$ directly to the regulation of Rho/ROCK signaling pathway were published [47-51]. PKC $\alpha$ regulates the localization of the small GTPase Rnd3 (also called RhoE) [47]. Rnd3 binds to ROCK kinase and prevents its downstream signaling upon Rnd3 binding, phosphorylation of MLCP is inhibited and formation of ROCK-induced stress fibers is disturbed [48]. Moreover, Rnd3 competes with PDK1 for binding to ROCK kinase and thus prevents its proper localization to the plasma membrane [49]. PKCa phosphorylates Rnd 3 and causes its translocation to internal membranes [47]. Upon this altered localization, Rnd3 is no longer able to inhibit the Rho/ROCK pathway, which is subsequently enhanced [47]. Moreover, Raf kinase pathway activation, which could be regulated by PKC $[50]$, was shown to be associated with Rnd3 expression [51].

Interestingly, in this study we have seen increased phosphorylation of cofilin on Ser-2 after ROCK inhibition. The positive effect of Y-27632 on cofilin phosphorylation was observed only in 3D suggesting that specific, and for AMT indispensable, condition of cultivation in 3D is responsible for the effect. Cofilin phosphorylation is regulated both by kinases (LIMK family) and phosphatases (SSH family). The KINEX microarray, however, allows to analyze only changes in expression and phosphorylation levels of LIMK1. It is hard to assess which of the pathways of cofilin phosphorylation is predominant in particular cells. We can speculate that in our case cofilin phosphorylation could be promoted via Rac/PAK1/LIMK2 signaling. In fact we see a mild $(60 \%)$ increase in PAK1 expression. This was not included in our results as the p Value $(0,079)$ did not meet our criteria for significance. Finally, though it might be contra intuitive others have also shown that Y27632 treatment can induce an increase in cofilin phosphorylation [52].

\section{Conclusions}

Our results show that PKC $\alpha$ is an important regulator of the amoeboid morphology of cancer cells and its downregulation causes AMT. Furthermore, our data also indicate that $\mathrm{PKC} \alpha$ is involved in both mesenchymal and amoeboid invasiveness, making it an attractive target for anti-metastatic therapies.

\section{Additional files}

Additional file 1: Figure S1. The effect of Y-27632 treatment on morphology of A375 m2 melanoma cells. Cells were grown in 3D collagen and after $24 \mathrm{~h}$ cell morphology was analyzed and documented using photomicroscopy. Left panel: A375 m2 cells; right panel: A375 m2 cells treated with $10 \mu \mathrm{M}$ Y-27632.

Additional file 2: Figure S2. The expression of PKC alpha variants and the effectiveness of PKCa silencing. A) The expression of wt PKCa, constitutively-active PKCa and dominant-negative PKCa in K2, MDA-MB-231 and A375m2 strains. Total PKCa and phosphorylation of PKCa at Thr497 were detected using corresponding antibodies in cell lysates from each strain expressing wt PKCa, constitutively-active PKCa and dominant-negative PKCa from pCMV6 vector. Representative immunoblots of each variant are shown. Actin was used as a loading control. B) The expression of total PKCa in siRNA-transfected A375m2 cells. Total PKCa level was detected in lysates from control cells (not transfected), mock cells (transfected only with transfection reagents, without siRNA) and siRNA transfected cells (siRNA against PKCa). Representative immunoblots are shown. Actin was used as a loading control. 
Additional file 3: Figure S3. The effect of PKCa activation with PMA and PKCa inhibition with Gö6976 on morphology of cancer cells. The effect of PKCa activation with PMA and PKCa inhibition with Gö6976 on morphology of cancer cells. Cells were grown in 3D collagen and after $24 \mathrm{~h}$ cell morphology was analyzed and documented using photomicroscopy. Top panels: K2 cells; middle panels: A375 m2 cells; bottom panels: MDA-MB-231 cells. Left panels: control untreated cells; medium panels: cells treated with $162 \mathrm{nM}$ PMA; right panels: cells treated with 1 MM Gö6976.

Additional file 4: Figure S4. The effect of PKCa variants expression on morphology of cancer cells. Cells were grown in 3D collagen and after $24 \mathrm{~h}$ cell morphology was analyzed and documented using photomicroscopy. Top panels: K2 cells; middle panels: A375 m2 cells; bottom panels: MDA-MB-231 cells. Left panels: control cells; medium panels: cells overexpressing wt PKCa; right panels: cells expressing dominant-negative version of $\mathrm{PKCa}$.

Additional file 5: Figure S5. The effect of PKCa silencing on morphology of A375 m2 melanoma cells. Cells were grown in 3D collagen and after $24 \mathrm{~h}$ cell morphology was analyzed and documented using photomicroscopy. Left panel: A375m2 cells; medium panel: A375m2 cells treated with scrambled siRNA; right panel: A375m2 cells treated with PKCa-specific siRNA.

Additional file 6: Figure S6. The effect of PKCa on gelatin degradation by $\mathrm{A} 375 \mathrm{~m} 2$ cells. The effect of PKCa on matrix-degrading activity in A375M2 melanoma cells. (A) Cells were plated on fluorescent gelatin-coated coverslips and subjected to gelatin degradation assay as described in section "Methods". Representative fields were documented by photomicroscopy. Left panel: A375M2 cells; middle panel: A375M2 cells treated with scrambled siRNA; right panel: A375M2 cells treated with PKCa -specific siRNA. Top panel: F-actin staining; bottom panel: gelatin degradation. (B) The quantification of matrix degrading activity was performed as described in section "Methods". Histogram bars represent mean relative degradation obtained from 3 independent experiments and normalized to that of untreated A375M2 cells, the error bars represents standard deviations. Statistical significance was evaluated according to unpaired two-tailed Student's t-test. Significant difference $(p<0.01)$ in comparison to mock-transfected cells is indicated by asterisks.

\section{Abbreviations}

AMT: Amoeboid-mesenchymal transition; ECM: Extracellular matrix MAT: Mesenchymal-amoeboid transition; MLC: Myosin light chain; MLCP: Myosin light chain phosphatase; PMA: Phorbol 12-myristate 13-acetate.

\section{Competing interests}

The authors declare that they have no competing interests.

\section{Authors' contributions}

$\mathrm{KV}$ : performed experiments with PKCa activators and inhibitors and helped to draft the manuscript. ES, AG: performed experiments with PKCa expression constructs. LK: performed the proteomic analysis of AMT in 3D. VC: performed the statistical analysis and helped to draft the manuscript. DR, JB: conceived of the study, and participated in its design and coordination and helped to draft the manuscript. All authors read and approved the final manuscript.

\section{Acknowledgements}

The work was supported by grants from the Kellner Family Foundation Principal Investigator Grant and by the project BIOCEV (CZ.1.05/1.1.00/ 02.0109) from the European Regional Development Fund and co-financed by the European Social Fund and the state budget of the Czech Republic (project no. CZ.1.07/2.3.00/30.0061)

\section{Author details}

'Department of Cell Biology, Laboratory of Cancer Cell Invasion, Charles University in Prague, Prague, Czech Republic. ${ }^{2}$ Current affiliation: Microscopy Unit, Institute of Experimental Medicine, The Czech Academy of Sciences, Prague, Czech Republic.
Received: 3 November 2014 Accepted: 22 April 2015

Published online: 29 April 2015

\section{References}

1. Kopfstein L, Christofori G. Metastasis: cell-autonomous mechanisms versus contributions by the tumor microenvironment. Cell Mol Life Sci. 2006;63(4):449-68.

2. Farooqui R, Fenteany G. Multiple rows of cells behind an epithelial wound edge extend cryptic lamellipodia to collectively drive cell-sheet movement. J Cell Sci. 2005;118(Pt 1):51-63.

3. Friedl P, Gilmour D. Collective cell migration in morphogenesis, regeneration and cancer. Nat Rev Mol Cell Biol. 2009;10(7):445-57.

4. Friedl P, Noble PB, Walton PA, Laird DW, Chauvin PJ, Tabah RJ, et al. Migration of coordinated cell clusters in mesenchymal and epithelial cancer explants in vitro. Cancer Res. 1995:55(20):4557-60.

5. Friedl P. Prespecification and plasticity: shifting mechanisms of cell migration. Curr Opin Cell Biol. 2004;16(1):14-23.

6. Pankova K, Rosel D, Novotny M, Brabek J. The molecular mechanisms of transition between mesenchymal and amoeboid invasiveness in tumor cells. Cell Mol Life Sci. 2010;67(1):63-71.

7. Nobes CD, Hall A. Rho, rac, and cdc42 GTPases regulate the assembly of multimolecular focal complexes associated with actin stress fibers, lamellipodia, and filopodia. Cell. 1995;81(1):53-62.

8. Ridley AJ, Paterson HF, Johnston CL, Diekmann D, Hall A. The small GTP-binding protein rac regulates growth factor-induced membrane ruffling. Cell. 1992;70(3):401-10.

9. Tolde $O$, Rosel D, Vesely P, Folk P, Brabek J. The structure of invadopodia in a complex 3D environment. Eur J Cell Biol. 2010;89(9):674-80.

10. Kimura K, Ito M, Amano M, Chihara K, Fukata Y, Nakafuku M, et al. Regulation of myosin phosphatase by Rho and Rho-associated kinase (Rho-kinase). Science. 1996:273(5272):245-8.

11. Mierke CT, Rosel D, Fabry B, Brabek J. Contractile forces in tumor cell migration. Eur J Cell Biol. 2008;87(8-9):669-76.

12. Sahai E, Marshall CJ. ROCK and Dia have opposing effects on adherens junctions downstream of Rho. Nat Cell Biol. 2002:4(6):408-15.

13. Rosel D, Brabek J, Tolde O, Mierke CT, Zitterbart DP, Raupach C, et al. Up-regulation of Rho/ROCK signaling in sarcoma cells drives invasion and increased generation of protrusive forces. Mol Cancer Res. 2008;6(9):1410-20.

14. Wyckoff JB, Pinner SE, Gschmeissner S, Condeelis JS, Sahai E. ROCK- and myosin-dependent matrix deformation enables protease-independent tumor-cell invasion in vivo. Curr Biol. 2006;16(15):1515-23.

15. Friedl P, Wolf K. Plasticity of cell migration: a multiscale tuning model. J Cell Biol. 2010;188(1):11-9.

16. Sanz-Moreno V, Marshall CJ. The plasticity of cytoskeletal dynamics underlying neoplastic cell migration. Curr Opin Cell Biol. 2010;22(5):690-6.

17. Wolf $\mathrm{K}$, Mazo I, Leung $\mathrm{H}$, Engelke $\mathrm{K}$, von Andrian UH, Deryugina El, et al. Compensation mechanism in tumor cell migration: mesenchymalamoeboid transition after blocking of pericellular proteolysis. J Cell Biol. 2003;160(2):267-77.

18. Janostiak $R$, Janoštiak $R$, Tolde $O$, Brůhová Z, Novotný M, Hanks SK, et al. Tyrosine phosphorylation within the $\mathrm{SH} 3$ domain regulates CAS subcellular localization, cell migration, and invasiveness. Mol Biol Cell. 2011;22(22):4256-67.

19. R Core Team. R: a language and environment for statistical computing Vienna, Austria: R Foundation for Statistical Computing; 2013. ISBN 3-900051-07-0.

20. Bornancin F, Parker PJ. Phosphorylation of protein kinase C-alpha on serine 657 controls the accumulation of active enzyme and contributes to its phosphatase-resistant state. J Biol Chem. 1997;272(6):3544-9.

21. Gysin S, Imber R. Replacement of Ser657 of protein kinase C-alpha by alanine leads to premature down regulation after phorbol-ester-induced translocation to the membrane. Eur J Biochem. 1996:240(3):747-50.

22. Nakashima S. Protein kinase C alpha (PKC alpha): regulation and biological function. J Biochem. 2002;132(5):669-75

23. Konopatskaya O, Poole AW. Protein kinase Calpha: disease regulator and therapeutic target. Trends Pharmacol Sci. 2010;31(1):8-14.

24. Du HF, Ou LP, Yang X, Song XD, Fan YR, Tan B, et al. A new PKCalpha/beta/ TBX3/E-cadherin pathway is involved in PLCepsilon-regulated invasion and migration in human bladder cancer cells. Cell Signal. 2014;26(3):580-93.

25. Hoshino D, Jourquin J, Emmons SW, Miller T, Goldgof M, Costello K, et al. Network analysis of the focal adhesion to invadopodia transition identifies a PI3K-PKCalpha invasive signaling axis. Sci Signal. 2012;5(241):2002964. 
26. Lin CC, Lee IT, Wu WB, Liu CJ, Hsieh HL, Hsiao LD, et al. Thrombin mediates migration of rat brain astrocytes via PLC, Ca(2)(+), CaMKII, PKCalpha, and AP-1-dependent matrix metalloproteinase-9 expression. Mol Neurobiol. 2013:48(3):616-30.

27. Shu Q, Hu ZL, Huang C, Yu XW, Fan H, Yang JW, et al. Orexin-A promotes cell migration in cultured rat astrocytes via Ca2 +-dependent PKCalpha and ERK1/2 signals. PLoS One. 2014;9(4):2014.

28. Wang C, Wang $X$, Liang $H$, Wang $T$, Yan $X$, Cao M, et al. miR-203 inhibits cell proliferation and migration of lung cancer cells by targeting PKCalpha. PLoS One. 2013:8(9):2013.

29. Wu B, Zhou H, Hu L, Mu Y, Wu Y. Involvement of PKCalpha activation in TFNlla/PAR2-induced proliferation, migration, and survival of colon cancer cell SW620. Tumour Biol. 2013:34(2):837-46.

30. Koivunen J, Aaltonen V, Koskela S, Lehenkari P, Laato M, Peltonen J. Protein kinase C alpha/beta inhibitor Go6976 promotes formation of cell junctions and inhibits invasion of urinary bladder carcinoma cells. Cancer Res. 2004;64(16):5693-701.

31. Masur K, Lang K, Niggemann B, Zanker KS, Entschladen F. High PKC alpha and low E-cadherin expression contribute to high migratory activity of colon carcinoma cells. Mol Biol Cell. 2001;12(7):1973-82.

32. Engers R, Mrzyk S, Springer E, Fabbro D, Weissgerber G, Gernharz CD, et al. Protein kinase $\mathrm{C}$ in human renal cell carcinomas: role in invasion and differential isoenzyme expression. Br J Cancer. 2000;82(5):1063-9.

33. Parsons M, Keppler MD, Kline A, Messent A, Humphries MJ, Gilchrist R, et al. Site-directed perturbation of protein kinase C- integrin interaction blocks carcinoma cell chemotaxis. Mol Cell Biol. 2002;22(16):5897-911.

34. Podar K, Tai YT, Lin BK, Narsimhan RP, Sattler M, Kijima T, et al. Vascular endothelial growth factor-induced migration of multiple myeloma cells is associated with beta 1 integrin- and phosphatidylinositol 3-kinasedependent PKC alpha activation. J Biol Chem. 2002;277(10):7875-81.

35. Hu JG, Wang XF, Zhou JS, Wang FC, Li XW, Lu HZ. Activation of PKC-alpha is required for migration of C6 glioma cells. Acta Neurobiol Exp. 2010;70(3):239-45.

36. Lonne GK, Cornmark L, Zahirovic IO, Landberg G, Jirstrom K, Larsson C. PKCalpha expression is a marker for breast cancer aggressiveness. Mol Cancer. 2010;9(76):1476-4598.

37. Haughian JM, Bradford AP. Protein kinase C alpha (PKCalpha) regulates growth and invasion of endometrial cancer cells. J Cell Physiol. 2009;220(1):112-8

38. Cameron AJ, Procyk KJ, Leitges M, Parker PJ. PKC alpha protein but not kinase activity is critical for glioma cell proliferation and survival. Int Cancer. 2008;123(4):769-79.

39. Wolf $\mathrm{K}$, Te Lindert $\mathrm{M}$, Krause $\mathrm{M}$, Alexander $\mathrm{S}$, Te Riet J, Willis AL, et al. Physical limits of cell migration: control by ECM space and nuclear deformation and tuning by proteolysis and traction force. J Cell Biol. 2013;201(7):1069-84.

40. Mehta D, Rahman A, Malik AB. Protein kinase C-alpha signals rho-guanine nucleotide dissociation inhibitor phosphorylation and rho activation and regulates the endothelial cell barrier function. J Biol Chem. 2001;276(25):22614-20.

41. Singh I, Knezevic N, Ahmmed GU, Kini V, Malik AB, Mehta D. GalphaqTRPC6-mediated Ca2+ entry induces RhoA activation and resultant endothelial cell shape change in response to thrombin. J Biol Chem. 2007;282(11):7833-43.

42. Holinstat M, Mehta D, Kozasa T, Minshall RD, Malik AB. Protein kinase Calpha-induced p115RhoGEF phosphorylation signals endothelial cytoskeletal rearrangement. J Biol Chem. 2003;278(31):28793-8.

43. Dovas A, Choi Y, Yoneda A, Multhaupt HA, Kwon SH, Kang D, et al. Serine 34 phosphorylation of rho guanine dissociation inhibitor (RhoGDlalpha) links signaling from conventional protein kinase $C$ to RhoGTPase in cell adhesion. J Biol Chem. 2010;285(30):23296-308.

44. DerMardirossian C, Bokoch GM. GDls: central regulatory molecules in Rho GTPase activation. Trends Cell Biol. 2005;15(7):356-63.

45. Avalos AM, Valdivia AD, Munoz N, Herrera-Molina R, Tapia JC, Lavandero S, et al. Neuronal Thy- 1 induces astrocyte adhesion by engaging syndecan- 4 in a cooperative interaction with alphavbeta3 integrin that activates PKCalpha and RhoA. J Cell Sci. 2009;122(Pt 19):3462-71.

46. Dovas A, Yoneda A, Couchman JR. PKCbeta-dependent activation of RhoA by syndecan-4 during focal adhesion formation. J Cell Sci. 2006;119(Pt 13):2837-46.
47. Madigan JP, Bodemann BO, Brady DC, Dewar BJ, Keller PJ, Leitges M, et al. Regulation of Rnd3 localization and function by protein kinase $C$ alpha-mediated phosphorylation. Biochem J. 2009;424(1):153-61.

48. Riento K, Guasch RM, Garg R, Jin B, Ridley AJ. RhoE binds to ROCK I and inhibits downstream signaling. Mol Cell Biol. 2003;23(12):4219-29.

49. Pinner S, Sahai E. PDK1 regulates cancer cell motility by antagonising inhibition of ROCK1 by RhoE. Nat Cell Biol. 2008;10(2):127-37.

50. Hwang YP, Yun HJ, Kim HG, Han EH, Lee GW, Jeong HG. Suppression of PMA-induced tumor cell invasion by dihydroartemisinin via inhibition of PKCalpha/Raf/MAPKs and NF-kappaB/AP-1-dependent mechanisms. Biochem Pharmacol. 2010;79(12):1714-26.

51. Hansen $\mathrm{SH}$, Zegers MM, Woodrow M, Rodriguez-Viciana $\mathrm{P}$, Chardin $\mathrm{P}$, Mostov KE, et al. Induced expression of Rnd3 is associated with transformation of polarized epithelial cells by the Raf-MEK-extracellular signal-regulated kinase pathway. Mol Cell Biol. 2000;20(24):9364-75.

52. Rochelle T, Daubon T, Van Troys M, Harnois T, Waterschoot D, Ampe C, et al. p210bcr-abl induces amoeboid motility by recruiting ADF/destrin through RhoA/ROCK1. FASEB J. 2013;27(1):123-34.

\section{Submit your next manuscript to BioMed Central and take full advantage of:}

- Convenient online submission

- Thorough peer review

- No space constraints or color figure charges

- Immediate publication on acceptance

- Inclusion in PubMed, CAS, Scopus and Google Scholar

- Research which is freely available for redistribution 